\title{
Graft reperfusion of transplanted liver-perfect storm for coronary vasospasm
}

\author{
Latha Hebbar*, Eric W Nelson, Gregory Scott Kottkamp and Sylvia H Wilson \\ Department of Anesthesia and Perioperative Medicine, Medical University of South Carolina, South Carolina, USA
}

\begin{abstract}
Major adverse cardiac events in patients during liver transplantation are due to preexisting cardiopulmonary comorbidities and the impact of surgery on hemodynamics. Graft reperfusion of the transplanted liver is the most hemodynamically critical phase and can be associated with air embolism and the release of acidotic, cold, hyperkalemic effluent containing vaso-constrictive chemical mediators such endothelin-1 and thromboxane A2. Concomitant release of oxygen reactive species can further sensitize the coronary vasculature to vasoconstrictors creating the perfect milieu for coronary vasospasm. We report a case in which myocardial ischemia accompanied by significant ST depression followed reperfusion possibly caused by coronary vasospasm.
\end{abstract}

\section{Introduction}

End-stage liver disease affects every organ system in the body leading to a very exhaustive preoperative workup to determine eligibility for a potential liver transplant. The AHA/ACA have established guidelines for cardiac workup of these patients, however, the frequency of surveillance while on the transplant waiting list is yet to be determined [1]. The anesthetic management of these cases is challenging with reperfusion of the transplanted graft being the most critical period. Hemodynamic changes observed during graft reperfusion include decreases in systemic vascular resistance (SVR) and mean arterial blood pressure (MAP), dysrhythmias, abrupt increases in mean PA pressure, CVP and PCWP, myocardial ischemia and cardiac arrest [2,3]. The causes of this spectrum of hemodynamic instability are multifactorial and complex. They include the sudden release of cold, acidotic, hyperkalemic preservation fluid along with vasoactive mediators into the systemic circulation, surgical anastomotic mishaps with accompanying blood loss and air embolism causing right ventricular outflow tract obstruction and failure [2-5].

Myocardial ischemia with accompanying ST-T wave changes can occur during graft reperfusion. An imbalance of myocardial oxygen supply vs demand due to systemic hypotension, profound anemia or paradoxical coronary air embolism could be contributory. We report a case of myocardial ischemia during liver reperfusion attributed to coronary vasospasm. The setting of graft reperfusion with associated decreases in core temperature, increases in endothelin-1 and thromboxane A2 levels and increases in reactive oxygen species (ROS) creates a perfect storm for this clinical phenomenon. This report describes the care of one patient who has provided us with written consent to disclose the management of his case.

\section{Case description}

Preoperative: A 66 year old Caucasian male with NASH cirrhosis, MELD score of 15, was scheduled for an OLTx. His PMH was significant for DM II and chronic kidney disease (CKD). His exercise tolerance was limited due to his advanced liver disease but he denied any history of chest pain or history of ischemic heart disease. His preoperative cardiac workup included serial ECHO's and CST-adenosine MPS. ECHO performed 3 years ago revealed $\mathrm{EF}$ of $64 \%$, normal valves and $\mathrm{RV}$ systolic of $43 \mathrm{mmHg}$. Bubble ECHO did not demonstrate any $\mathrm{R}$ to $\mathrm{L}$ or $\mathrm{L}$ to $\mathrm{R}$ intracardiac shunting or intrapulmonary shunting. CST-adenosine MPS two years ago showed an EF of 63\%, no reversible ischemia but a fixed defect was noted at the apex. No RWMA was noted on ECHO and a bubble study was not performed as his RA saturation was $100 \%$. The immediate pretransplant CST-adenosine MPS demonstrated decrease perfusion at anterospetal and apical walls with fixed defects at the anteroseptal wall, EF of 59\%, no RWMA and RV systolic of $49 \mathrm{mmHg}$. His preoperative EKG was normal sinus rhythm with no ST-T wave abnormalities.

Intraop: The patient underwent an uneventful anesthetic induction and line placement, which included two antecubital 8.5 Fr RIC lines, two 9 Fr Cordis sheaths and a radial arterial line with continuous cardiac output monitoring which is standard practice at our institution. Maintenance of anesthesia included isoflurane, cis-atracurium and sufentanil infusion. According to institutional protocol, the patient was also on a calcium chloride infusion at $1 \mathrm{gm} / \mathrm{hr}$. Significant portal hypertension required an octreotide $(25 \mathrm{mcg} / \mathrm{hour}$ ) infusion and transfusion of blood products during the dissection phase. With a history of CKD, increasing serum potassium levels necessitated the use of washed PRBC transfusions. The octreotide infusion was discontinued at the commencement of the anhepatic phase.

Anhepatic/Reperfusion: A piggy-back technique was used for graft anastomosis which was hemodynamically tolerated by the patient. The donor liver was flushed via the portal vein with 2 liters of Plasmalyte containing $25 \mathrm{~g}$ of albumin during the anhepatic phase. Pre-reperfusion lab values included K 4.2, Hct 28, BE -9, ionized Ca 1.16 and INR 1.4.

Correspondence to: Latha Hebbar, Department of Anesthesia and Perioperative Medicine, Medical University of South Carolina, South Carolina, USA, E-mail: hebbarl@musc.edu

Received: November 08, 2016; Accepted: December 24, 2016; Published: December 28, 2016 
In preparation for reperfusion, the patient was hyperventilated with an $\mathrm{FiO}_{2}$ of 1.0 to an $\mathrm{ETCO}_{2}$ of 28 and the following medications were administered: $50 \mathrm{mEq}$ sodium bicarbonate, 1 gram of calcium chloride and $100 \mathrm{mg}$ of lidocaine. With a history of rising potassium levels, a blood flush and non-systemic reperfusion was performed. EKG immediately following reperfusion indicated NSR with mild peaking of T waves, An additional 2 grams of Calcium chloride was administered and the $\mathrm{T}$ waves returned to normal. Immediate post reperfusion, pressor support included phenylephrine at $40 \mathrm{mcg} / \mathrm{min}$ and vitals were MAP 65-70 mmHg, HR 60-65 bpm, CI 3.3, CVP $8 \mathrm{mmHg}$ and core temperature $36.8 \mathrm{deg} \mathrm{C}$.

Post reperfusion: 11 minutes post reperfusion $A B G$ showed a $\mathrm{K}$ 4.6, ionized calcium of 1.68 and Hct 26. CI was 3.2, MAP 60-65 mmHg and HR 60-65 bpm. At this time, ST segment depression was noted in leads II, II and avF which progressed from $-0.5 \mathrm{~mm}$ to a maximum of $4.2 \mathrm{~mm}$ over 15 minutes (Figure 1). Transesophageal echocardiography (TEE), done at the time of severe ST changes revealed global hypokinesis with an ejection fraction (EF) of $20 \%$. There was severe wall motion abnormality in the anterior and inferior walls, worse at the apex than the base. RV function was normal and no intracardiac air was noted. All valve functions were normal.

Epinephrine infusion was started at $0.25 \mathrm{mcg} / \mathrm{kg} / \mathrm{min}$ to maintain $\mathrm{CI}$ and metoprolol $1 \mathrm{mg}$ was given which maintained MAP between 65-70 and HR at 60-65 bpm with NSR. Since the TEE findings did not demonstrate intracardiac air and given the fact that a pre-op bubble ECHO was negative for both cardiac and intrapulmonary shunting, paradoxical air embolism to the coronary arteries was ruled out and coronary vasospasm was suspected. A bolus dose of $50 \mathrm{mcg}$ nitroglycerin was administered and troponin levels were sent. Cardiology was contacted and while the decision for further management was being discussed, the ST segment depression started to improve and normalized within the next 15 mins (Figure 2). TEE with concomitant normalization of the ST segments demonstrated a wall

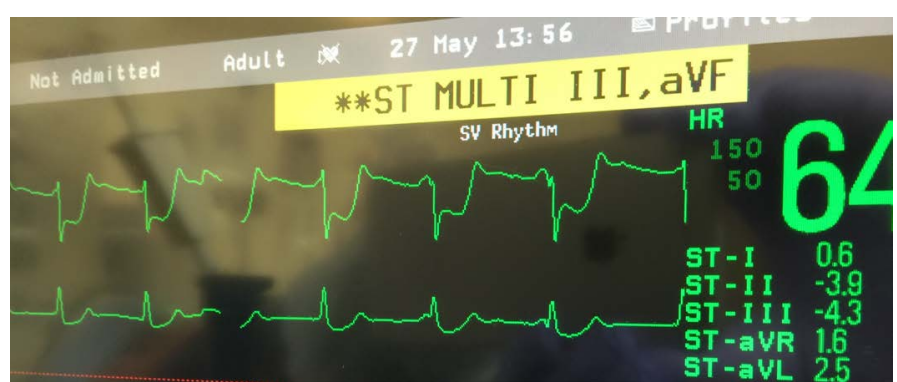

Figure 1. EKG changes 26 minutes after reperfusion showing maximal ST depression. ST segment depression of -3.9 in Lead II and -4.3 in Lead III is noted. Lead aVF (not seen in figure) also had significant ST depression.

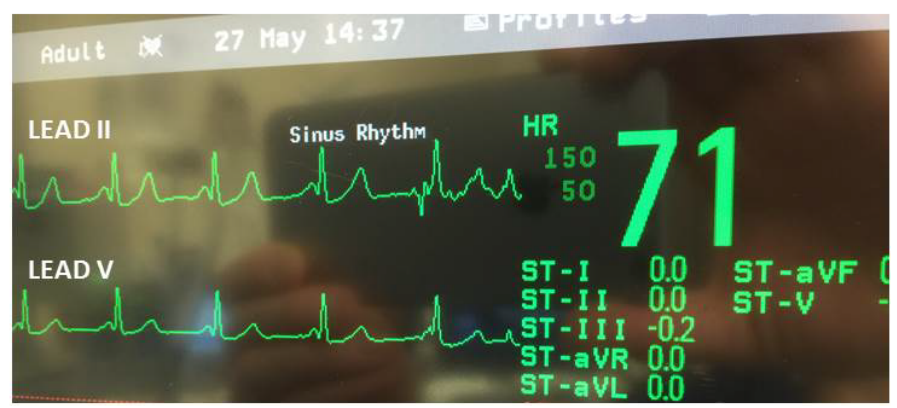

Figure 2. EKG 60 minutes following reperfusion showing normalization of the ST segment in Lead II and III and aVF (not shown). motion abnormality in the anterior and inferior walls; however, it was less severe. The other walls of the heart had normal function and the EF returned to $40 \%$. The patient required epinephrine infusion at 0.25 $\mathrm{mcg} / \mathrm{kg} / \mathrm{min}$ for a further $45 \mathrm{mins}$ and then was weaned off all pressors. The remainder of the case proceeded uneventfully and patient was transferred intubated to the ICU, where he was extubated the following day. The patient's serial troponin levels peaked at 0.79 on POD 1 and normalized by POD 3. TTE on POD 1 revealed an EF of 54\% with focal apical hypokinesis. Since the patient was asymptomatic, left heart catheterization was postponed until complications related to the bile duct anastomosis were resolved.

\section{Discussion}

Catastrophic and potentially fatal hemodynamic changes can occur during reperfusion of the transplanted liver. Optimal management demands a rapid and accurate diagnosis. Post reperfusion syndrome (PRS), defined as $>30 \%$ decrease in mean arterial pressure for at least 1 min during the first 5 minutes after reperfusion, is seen in $30-40 \%$ of patients [2,3]. The etiology of PRS is complex and multifactorial. Pathophysiology of the hemodynamic instability could be related to deceases in preload, afterload, myocardial contractility of either/both ventricles or rate and/or rhythm changes [4]. These cardiovascular alterations result from a complex phenomenon possibly related to sudden release of cold, acidotic, and hyperkalemic preservation fluid along with vasoactive mediators into the circulation [5]. Myocardial ischemia occurs when there is inadequate oxygen supply commensurate with oxygen demand. Paradoxical air embolus as a cause of myocardial ischemia during reperfusion has been described. [6] Coronary artery spasm (CAS) is a well-established cause of myocardial ischemia and occurs from the interplay of 2 factors - 1) localized/diffuse abnormality of a coronary artery which sensitizes it to vasoconstrictor stimuli and 2) a vasoconstrictor stimulus which can provoke spasm $[7,8]$. Vasoconstrictor stimuli include catecholamines, acetylcholine, histamine, serotonin, vasopressin, thromboxane A2, endothelin-1, thrombin and alkalosis [7]. CAS is response to a cold pressor test has also been described [9].

In the setting of a graft reperfusion, some of the vasoconstrictor stimuli include decrease in core temperature, thrombin and respiratory alkalosis from overzealous ventilation in anticipation of reperfusion. There has been one case report of possible coronary vasospasm during liver transplant attributed to core hypothermia [10].

The role of vasoactive mediators as vasoconstrictive stimuli in this setting needs to be highlighted. Endothelin-1, a potent vasoconstrictive peptide, has been shown in animal models to be released during liver reperfusion $[11,12]$. Endothelin-1 has been shown to play an essential role in ROS-dependent coronary vasospasm in the setting of endothelial dysfunction [13]. Levels of thromboxane A2, another vasoconstrictive peptide, is also increased during graft reperfusion [14]. An increase in ROS has been demonstrated with reperfusion of the liver and this has a detrimental effect, causing both endothelial dysfunction and inflammation [15]. Increases in oxygen reactive species also sensitizes the constrictor response of some the vasoconstrictive stimuli [7].

Our patient, though asymptomatic preoperatively, had baseline coronary artery disease as evidence by the preoperative MPS. Myocardial ischemia from increases in oxygen demand was not considered as the patient had heart rate near baseline of 65-70 bpm. Oxygen supply due to anemia was also ruled out. Paradoxical air embolism as a differential diagnosis was considered; however, there was no TEE evidence of intra-cardiac air and preoperative testing 
was negative for intrapulmonary and cardiac shunting. The transient nature of the intraoperative ST segment depression and return to baseline with nitroglycerine made coronary vasospasm a possible cause. Thrombin as a cause of vasospasm was ruled out due to the fact that the patients INR post reperfusion was 2.7. Arterial blood gases did not reflect alkalosis as a contributory factor for vasospasm. Since there was no drop in core temperature, hypothermia could not be considered as contributing to the CAS. Octreotide, a potential coronary vasoconstrictor was discontinued during the anhepatic phase and could not be implicated in causing CAS. Vasoactive mediators, endothelin-1 and thromboxane A2 in the setting of an increase ROS environment related to graft reperfusion may have played a significant role, especially with a history of MPS demonstrated CAD.

In conclusion, graft reperfusion of the transplanted liver creates a perfect storm for the development of coronary vasospasm and should be included in the plethora of hemodynamic events that can occur during this critical period, especially in vulnerable patients. Early recognition and rapid diagnosis of coronary vasospasm is critical for successful management. TEE is associated with some degree of morbidity in these patients; however, the benefits certainly outweigh the risks and should be considered a vital monitoring tool for liver transplantation surgery.

\section{References}

1. Lentine KL, Costa SP, Weir MR, Robb JR, Fleisher LA, et al. (2012) on behalf of the American Heart Association Council on the Kidney in Cardiovascular Disease Research and Council on Peripheral Vascular Disease. Cardiac disease evaluation and management among kidney and liver transplantation candidates: A scientific statement from the American Heart Association and the American College of Cardiology Foundation. J Am Coll Cardiol 126: 617-663. [Crossref]

2. Aggarwal S, Kang Y, Freeman JA (1987) Postreperfusion syndrome: Cardiovascular collapse following hepatic reperfusion during liver transplantation. Transplant Proc 19: 54-55. [Crossref]

3. Paugam-Burtz C, Kavafyan J, Merckx P (2009) Postreperfusion syndrome during liver transplantation for cirrhosis: Outcome and predictors. Liver Transpl 15: 522-529. [Crossref]
4. Ayhan A, Araz C, Komurcu O, Kaplan S, Torgay A, et al. (2015) The Evaulation of hemodynamic changes during the reperfusion phase in adult liver donor liver transplantations: the role of cardiovascular problems. Transplant Proc 47: 1199-203. [Crossref]

5. Nanashima A, Pillay P, Crawford M (2001) Analysis of postrevascularization syndrome after orthotopic liver transplantation: The experience of an Australian liver transplantation center. J Hepato Biliary Pancreat Surg 8: 557-563. [Crossref]

6. Kinscherff DM, Picton P, Kollars J, Dorje P (2010) Transjugular intrahepatic portosystemic shunt related to paradoxical air embolism during orthotopic liver transplantation. Can J Anaesth 57: 185-186. [Crossref]

7. Lanza GA, Careri G, Crea F (2011) Contemporary reviews in Cardiovascular Medicine. Mechanisms of Coronary Artery Spasm. Circulation 124: 1744-1782.

8. Kaski JC, Crea F, Meran D, Rodriguez L, Araujo L et al. (1986) Local coronary supersensitivity to diverse vasoconstrictive stimuli in patients with variant angina. Circulation 74: 1255-65. [Crossref]

9. Shea DJ, Ockene IS, Greene HL (1981) Acute myocardial infarction provoked by a cold pressor test. Chest 80: 649-651. [Crossref]

10. Ramsay M, Takaoka F, Brown M, Rosenlof P (1989) Coronary Artery Vasospasm following placement of a cold liver graft during orthotopic liver transplantation. Anesth Analg 69: 854-855. [Crossref]

11. Goto M, takei Y, Kawano S, Nagano K, Tsuji S et al. (1994) Endothelin-1 is involved in the pathogenesis of ischemia/reperfusion liver injury by hepatic microcirculatory disturbances. Hepatology 19: 675-81. [Crossref]

12. Charrueau CA, Carayon A, Thurman RG (2002) Long-term cold liver storage induces endothelin-1 release and a time-dependent increase in portal pressure at reperfusion in the rat. J Gastroenterol 37: 717-725. [Crossref]

13. Saitoh S, Matsumoto K, Kamioka M, Phkawara H, Kaneshiro T, et al. (2009) Nove pathway of endothelin-1 and reactive oxygen species in coronary vasospasm with endothelial dysfunction. Coron Artery Dis 20: 400-8. [Crossref]

14. Abu-Amara M, Yang M, Tapuria Y, Fuller B, Davidson B, et al. (2010) " Liver ischemia/reperfusion injury: processes in inflammatory networks - a review". Liver Transpl 16: 1016-1032. [Crossref]

15. Jaeschke H (2003) Molecular mechanisms of hepatic ischemia-reperfusion injury and preconditioning. Am J Physiol Gastrointest Liver Physiol 284: 15-26. [Crossref]

Copyright: (C2016 Ahmed SM. This is an open-access article distributed under the terms of the Creative Commons Attribution License, which permits unrestricted use, distribution, and reproduction in any medium, provided the original author and source are credited. 